\title{
Boundary behavior and rigidity of semigroups of holomorphic mappings
}

\author{
Mark Elin \\ Department of Mathematics \\ ORT Braude College \\ P.O. Box 78, 21982 Karmiel, Israel \\ e-mail: mark_elin@braude.ac.il \\ David Shoikhet \\ Department of Mathematics \\ ORT Braude College \\ P.O. Box 78, 21982 Karmiel, Israel \\ e-mail: davs@braude.ac.il
}

November 4, 2018

\begin{abstract}
In this paper we give some quantative characteristics of boundary asymptotic behavior of semigroups of holomorphic self-mappings of the unit disk including the limit curvature of their trajectories at the boundary Denjoy-Wolff point. This enable us to establish an asymptotic rigidity property for semigroups of parabolic type.
\end{abstract}

Key words and phrases: holomorphic mapping, asymptotic behavior, one-parameter semigroup, limit curvature, rigidity.

2000 Mathematics Subject Classification: 30C45, 47H20

\section{Introduction and main results}

We denote by $\operatorname{Hol}(D, \mathbb{C})$ the set of all holomorphic functions on a domain $D \subset \mathbb{C}$, and by $\operatorname{Hol}(D)$ the set of all holomorphic self-mappings of $D$. 
We say that a family $S=\left\{F_{t}\right\}_{t>0} \subset \operatorname{Hol}(D)$ is a one-parameter continuous semigroup on $D$ (semigroup, in short) if

(i) $F_{t}\left(F_{s}(z)\right)=F_{t+s}(z)$ for all $t, s \geq 0$ and $z \in D$, and

(ii) $\lim _{t \rightarrow 0^{+}} F_{t}(z)=z$ for all $z \in D$.

In the case when $D$ is the open unit disk $\Delta=\{z:|z|<1\}$, it follows from a result of E. Berkson and $\mathrm{H}$. Porta [1] that each semigroup is differentiable with respect to $t \in \mathbb{R}^{+}=[0, \infty)$. So, for each oneparameter continuous semigroup $S=\left\{F_{t}\right\}_{t \geq 0} \subset \operatorname{Hol}(\Delta)$, the limit

$$
\lim _{t \rightarrow 0^{+}} \frac{z-F_{t}(z)}{t}=f(z), \quad z \in \Delta,
$$

exists and defines a holomorphic mapping $f \in \operatorname{Hol}(\Delta, \mathbb{C})$. This mapping $f$ is called the (infinitesimal) generator of $S=\left\{F_{t}\right\}_{t \geq 0}$. Moreover, the function $u(t, z):=F_{t}(z),(t, z) \in \mathbb{R}^{+} \times \Delta$, is the unique solution of the Cauchy problem

$$
\left\{\begin{array}{l}
\frac{\partial u(t, z)}{\partial t}+f(u(t, z))=0, \\
u(0, z)=z, \quad z \in \Delta .
\end{array}\right.
$$

In the same paper, Berkson and Porta proved that a function $f \in$ $\operatorname{Hol}(\Delta, \mathbb{C})$ is a semigroup generator if and only if there are a point $\tau \in \bar{\Delta}$ and a function $p \in \operatorname{Hol}(\Delta, \mathbb{C})$ with $\operatorname{Re} p(z) \geq 0$, such that

$$
f(z)=(z-\tau)(1-z \bar{\tau}) p(z) .
$$

This representation is unique. Moreover, if $S$ contains neither the identity mapping nor an elliptic automorphism of $\Delta$, then $\tau$ is a unique attractive fixed point of $S$, i.e., $\lim _{t \rightarrow \infty} F_{t}(Z)=\tau, z \in \Delta$, and $\lim _{r \rightarrow 1^{-}} F_{t}(r \tau)=\tau$. The point $\tau$ is called the Denjoy-Wolff point of $S$.

In this paper we are interested in the boundary case $(\tau \in \partial \Delta)$ for which the asymptotic behavior of the semigroup has entirely different features then in the interior case. It was shown in [9] that if $\tau \in \partial \Delta$, then the angular derivative $f^{\prime}(\tau)=\angle \lim _{z \rightarrow \tau} f^{\prime}(z)=\angle \lim _{z \rightarrow 1} \frac{f(z)}{z-\tau}$ of $f$ at the point $\tau \in \partial \Delta$ exists and is a non-negative real number. One distinguishes two cases: (a) $f^{\prime}(\tau)>0$ (the hyperbolic case), and (b) $f^{\prime}(\tau)=0$ (the parabolic case).

Although the asymptotic behavior of semigroups has been studied by many mathematicians, the local geometry of semigroup trajectories 
near the boundary Denjoy-Wolff point have been attracting an intensive attention only recently.

It was shown that in many situations (in particular, always in the hyperbolic case) the semigroup trajectories have tangent lines passing through the Denjoy-Wolff point. Also, there is an essential difference between hyperbolic and parabolic type semigroups. Specifically, in the hyperbolic case the limit tangent line depends on the initial point of the trajectory, while in the parabolic case all the trajectories have the same tangent line (if it exists). See Fig. 1 and [3, ㅇ, 13, 5, 11] for details.

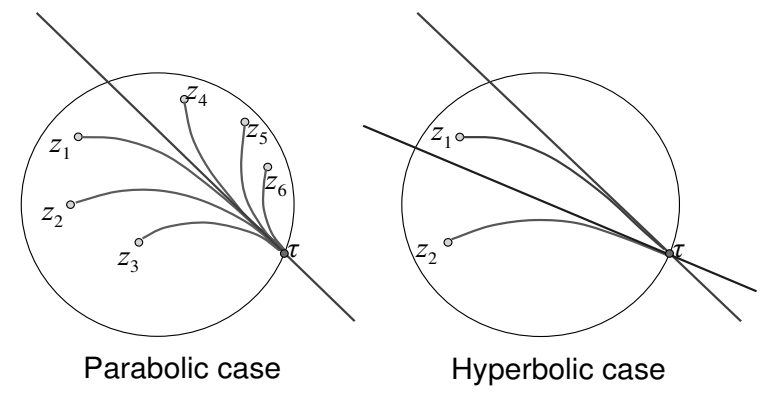

An advanced question in this study is: how close is a semigroup trajectory to its tangent line? In particular, one can ask: Is there a circle having the same tangent line, such that the trajectories lie between this circle and the line? See Fig. 2.

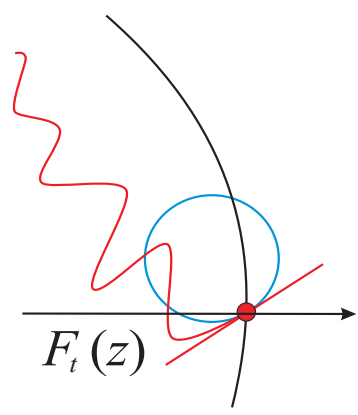

Since any trajectory $\gamma_{z}=\left\{F_{t}(z), t \geq 0\right\}, z \in \Delta$, is an analytic curve, it has a finite curvature at each its point $F_{s}(z)$. More precisely, given $z \in \Delta$ we denote by $\kappa(z, s)$ the curvature of the trajectory $\gamma_{z}$ at the point $F_{s}(z)$ and by $\kappa(z)$ the limit curvature of the trajectory: $\kappa(z):=$ $\lim _{s \rightarrow \infty} \kappa(z, s)$ (if it exists). Therefore, the above question is equivalent to 
the following one: When the limit curvature of a semigroup trajectory exists finitely?

This question is closely connected with the so-called asymptotic rigidity problem. Namely, one says that two semigroups with the same Denjoy-Wolff point $\tau \in \partial \Delta$ have a similar asymptotic behavior if

$$
\lim _{t \rightarrow \infty}\left(\arg \frac{1-\bar{\tau} F_{t}(z)}{1-\bar{\tau} G_{t}(z)}\right)=0 .
$$

From now on, we assume without loss of generality that $\tau=1$.

It turns out (see [8]) that two hyperbolic type semigroups having similar asymptotic behavior actually coincide up to rescaling. This fact is not longer true for parabolic type semigroups. Moreover, if the generator $f$ of a semigroup $S=\left\{F_{t}\right\}_{t \geq 0}$ admits the representation

$$
f(z)=b(z-1)^{2}+o\left((z-1)^{2}\right)
$$

then for each $z \in \Delta$, the limit tangent line to the trajectory $\gamma_{z}=$ $\left\{F_{t}(z), t \geq 0\right\}$ exists with

$$
\lim _{t \rightarrow \infty} \arg \left(1-F_{t}(z)\right)=-\arg b
$$

hence, does not depend on $z \in \Delta$ as well as on the remainder $o\left((z-1)^{2}\right)$ (see [8] and Theorem 1.4 (i) below).

Therefore, a natural conjecture is: If two semigroups having similar asymptotic behavior and the same limit curvatures, then they coincide up to rescaling. The proof of this conjecture follows directly from a more general fact (see Theorem 1.5 below).

To answer the questions above for a semigroup $S=\left\{F_{t}\right\}_{t \geq 0}$ generated by $f \in \operatorname{Hol}(\Delta, \mathbb{C})$, we apply a linearization model given by Abel's functional equation

$$
h\left(F_{t}(z)\right)=h(z)+t .
$$

It is rather simple to see that the function $h: \Delta \mapsto \mathbb{C}$ defined by

$$
h^{\prime}(z)=-\frac{1}{f(z)}, \quad h(0)=0,
$$

solves equation (1.3). This function is univalent and convex in the positive direction of the real axis due to (1.3). Sometimes it is called the Kœnigs function for the semigroup (see [3, ㅇ, 13, 17] and [11]). 
It is more or less known that in the hyperbolic case each semigroup trajectory $\gamma_{z}=\left\{F_{t}(z), t \geq 0\right\}$ converges to the boundary fixed point $\tau=1$ non-tangentially, as $t \rightarrow \infty$. The following quantitative result in this direction is given in 8 .

Theorem 1.1 Let $S=\left\{F_{t}\right\}_{t \geq 0}$ be a semigroup of hyperbolic type with the Denjoy-Wolff point $\tau=1$ and let $f$ be its generator with $f(1)=0$ and $a=f^{\prime}(1)>0$. Then

$$
\lim _{t \rightarrow \infty} \arg \left(1-F_{t}(z)\right)=a\left[\lim _{r \rightarrow 1^{-}} \operatorname{Im} h(r)-\operatorname{Im} h(z)\right] .
$$

In the present paper we first complete Theorem 1.1 by the following assertion.

Theorem 1.2 Let $S=\left\{F_{t}\right\}_{t \geq 0}$ be a semigroup of hyperbolic type with the Denjoy-Wolff point $\tau=1$ and let $f$ be its generator. Suppose that

$$
f(z)=a(z-1)+b(z-1)^{2}+R(z) \text {, where } \angle \lim _{z \rightarrow 1} \frac{R(z)}{(z-1)^{2}}=0 .
$$

Then for any initial point $z_{0} \in \Delta$ the limit curvature $\kappa\left(z_{0}\right)$ exists finitely. Moreover, the limit curvature circle can be defined by the following equality

$$
|1-z|^{2} \operatorname{Im}(b \bar{B})+a \operatorname{Im}((1-z) B)=0,
$$

where $B=\exp \left(a\left(h\left(z_{0}\right)-i \lim _{r \rightarrow 1^{-}} \operatorname{Im} h(r)\right)\right)$. In particular, the limit curvature is zero if and only if $\operatorname{Im}(b \bar{B})=0$.

The parabolic case is more delicate. In this situation there are semigroups which converge to the boundary Denjoy-Wolff point tangentially as well as examples of non-tangentially converging semigroups (see [3, 4, [5, 8]).

M. D. Contreras and S. Díaz-Madrigal in 3 have considered the set Slope $^{+}\left(\gamma_{z}\right)$ of all accumulation points (as $t \rightarrow \infty$ ) of the function $\arg \left(1-F_{t}(z)\right)$ and proved that these sets coincide for all $z \in \Delta$. In addition, they have proven that if for a function $h$ defined by (1.4), the image $h(\Delta)$ lies in a horizontal half-plane, then all the trajectories $\gamma_{z}$ tends tangentially to $\tau=1$. In addition, $\operatorname{Slope}^{+}\left(\gamma_{z}\right)$ is a single point which is equal to either $\pi / 2$ or $-\pi / 2$. In general the question whether Slope $^{+}\left(\gamma_{z}\right)$ is a singlton is still open (see [3, 13]). 
Inasmuch as we are updated, all results known in this direction require some smoothness conditions at the Denjoy-Wolff point. For example, if the semigroup generator $f$ is twice differentiable at the boundary DenjoyWolff point $\tau=1$, then all the trajectories $\left\{F_{t}(z), t \geq 0\right\}$ converge to this point tangentially if and only if $\operatorname{Re} f^{\prime \prime}(1)=0$ (see [8]).

Furthermore, it may happen that for each $z \in \Delta$, there is a horodisk $D(\tau, k):=\{\zeta \in \Delta: d(\zeta, \tau)<k\}, k=k(z)$, internally tangent to the unit circle at the point $\tau$, such that the trajectory $\left\{F_{t}(z)\right\}_{t \geq 0}$ lies outside $D(\tau, k)$. In this case we say that the semigroup $S$ converges to $\tau$ strongly tangentially. It is clear that the supremum of radii of such horodisks coincides with the limit curvature radius. Conversely, if a semigroup converges tangentially but not strongly tangentially, then its trajectories have infinite limit curvature.

For three times differentiable generators at the boundary DenjoyWolff point $\tau$ with $f^{\prime \prime \prime}(\tau)=0$ the following rigidity phenomenon was established in [16]: The semigroup $S$ generated by $f$ converges to $\tau$ strongly tangentially if and only if it consists of parabolic automorphisms of $\Delta$ (i.e., its trajectories have finite curvature).

Actually, the question on the finiteness of the limit curvature is a general problem which is also relevant for non-tangentially converging semigroups. In addition, one can ask: whether $\kappa(z)$ might be finite for some points $z \in \Delta$ and infinite to others? The following theorem answers these questions for semigroups generated by functions which are $(3+\varepsilon)$ smooth at the Denjoy-Wolff point.

Theorem 1.3 Let $S=\left\{F_{t}\right\}_{t \geq 0}$ be a semigroup generated by $f \in \mathcal{C}^{3+\varepsilon}(1)$, i.e.,

$$
f(z)=b(z-1)^{2}+c(z-1)^{3}+R(z),
$$

where $R \in \operatorname{Hol}(\Delta, \mathbb{C}), \lim _{z \rightarrow 1} \frac{R(z)}{(z-1)^{3+\varepsilon}}=0$, and let $b \neq 0$.

(a) If $\operatorname{Im} \frac{c}{b^{2}} \neq 0$, then all of the trajectories have infinite limit curvature, i.e., $\kappa\left(z_{0}\right)=\infty$ for each $z_{0} \in \Delta$.

(b) Otherwise, if $\operatorname{Im} \frac{c}{b^{2}}=0$, then each trajectory $\left\{F_{t}\left(z_{0}\right), t \geq 0\right\}$ has a finite limit curvature, namely, $\kappa\left(z_{0}\right)=\left|\frac{2 C}{b}\right|$, where

$$
C=|b|^{2} \operatorname{Im} h\left(z_{0}\right)+\operatorname{Im} b+\int_{0}^{\infty} \operatorname{Im}\left(\frac{f\left(F_{s}(1)\right) \bar{b}}{\left(1-F_{s}(0)\right)^{2}}-\frac{c \bar{b}}{b(s+1)}\right) d s .
$$


Thus, under above assumptions if $\kappa(z)$ is finite for some $z \in \Delta$, then it must be finite for all $z \in \Delta$.

Comparing Theorems 1.2 and 1.3 , we see again a cardinal difference between semigroups of hyperbolic and parabolic types: in the hyperbolic case with some smoothness conditions the limit curvature is always finite while in the parabolic case the limit curvature may be infinite. At the same time, it follows from [16] that if the second derivative $f^{\prime \prime}(1)$ is purely imaginary, then the third derivative $f^{\prime \prime \prime}(1)$ should be real. So, an immediate consequence of part (b) of Theorem 1.3 is the following fact.

Corollary 1.1 Let $\left\{F_{t}\right\}_{t>0}$ be a semigroup of holomorphic self-mappings of the open unit disk $\Delta$ generated by $f \in \mathcal{C}^{3+\varepsilon}(1)$ of the form (1.8) with $b \neq 0$. If $\operatorname{Re} b=0$, then each semigroup trajectory converges to $\tau=1$ strongly tangentially.

As a matter of fact, Theorem 1.3 is based on the following general result which contains complete quantitative characteristics of the asymptotic behavior for semigroups generated by functions smooth enough at the boundary Denjoy-Wolff points.

Theorem 1.4 Let $S=\left\{F_{t}\right\}_{t \geq 0}$ be a continuous semigroup of holomorphic self-mappings of the open unit disk $\Delta$ and let $f$ be its generator.

(i) Suppose that $f$ admits the following representation:

$$
f(z)=b(z-1)^{2}+R(z)
$$

where $R \in \operatorname{Hol}(\Delta, \mathbb{C}), \lim _{z \rightarrow 1} \frac{R(z)}{(z-1)^{2}}=0$. Then

$$
\frac{1}{1-F_{t}(z)}=-b t+G(z, t), \quad \text { where } \lim _{t \rightarrow \infty} \frac{G(z, t)}{t}=0
$$

and

$$
\lim _{t \rightarrow \infty}\left(\frac{1}{1-F_{t}(z)}-\frac{1}{1-F_{t}(0)}\right)=-b h(z) .
$$

(ii) If $b \neq 0$ and $R$ in (1.9) is of the form $R(z)=c(z-1)^{3}+R_{1}(z)$ with $R_{1} \in \operatorname{Hol}(\Delta, \mathbb{C}), \lim _{z \rightarrow 1} \frac{R_{1}(z)}{(z-1)^{3}}=0$, i.e., $f$ admits the representation:

$$
f(z)=b(z-1)^{2}+c(z-1)^{3}+R_{1}(z)
$$


then

$$
\frac{1}{1-F_{t}(z)}=-b t-\frac{c}{b} \log (t+1)+G_{1}(z, t),
$$

where $\lim _{t \rightarrow \infty} \frac{G_{1}(z, t)}{\log (t+1)}=0$, and

$$
\lim _{t \rightarrow \infty} t\left(\frac{1}{1-F_{t}(z)}-\frac{1}{1-F_{t}(0)}+b h(z)\right)=-\frac{c}{b} h(z) .
$$

(iii) If function $R_{1}$ in (1.12) satisfies the condition $\lim _{z \rightarrow 1} \frac{R_{1}(z)}{(z-1)^{3+\varepsilon}}=0$ for some $\varepsilon>0$, then there is a constant $A$ such that

$$
\frac{1}{1-F_{t}(z)}=-b t-\frac{c}{b} \log (t+1)-b h(z)+A+G_{2}(z, t),
$$

where $\lim _{t \rightarrow \infty} G_{2}(z, t)=0$.

Assertion (i) of Theorem 1.4 was proven in [8]. For the sake of completeness we prove it below in another way.

Now we are at the point to formulate our rigidity result which is also a consequence of Theorem 1.4.

Theorem 1.5 Let $\left\{F_{t}\right\}_{t>0},\left\{G_{t}\right\}_{t \geq 0} \subset \operatorname{Hol}(\Delta)$ be two continuous semigroups of holomorphic self-mappings of the open unit disk $\Delta$ generated by mapping $f$ and $g$, respectively. Suppose that $f$ and $g$ admit the following representations:

$$
\begin{gathered}
f(z)=b(z-1)^{2}+c_{1}(z-1)^{3}+r_{1}(z), \\
g(z)=b(z-1)^{2}+c_{2}(z-1)^{3}+r_{2}(z),
\end{gathered}
$$

where $\lim _{z \rightarrow 1} \frac{r_{1}(z)}{(z-1)^{3+\varepsilon}}=\lim _{z \rightarrow 1} \frac{r_{2}(z)}{(z-1)^{3+\varepsilon}}=0$. If

$$
\lim _{t \rightarrow \infty} \operatorname{Im}\left[\bar{b}\left(\frac{1}{1-F_{t}(z)}-\frac{1}{1-G_{t}(z)}\right)\right]=0
$$

then the semigroups coincide.

In the particular case when the limit curvature is finite, we affirm the asymptotic rigidity conjecture mentioned above.

Corollary 1.2 If the trajectories of two parabolic type semigroups generated by mappings of the class $\mathcal{C}^{3+\varepsilon}(1)$, have the same limit curvature circles, then the semigroups coincide up to rescaling. 


\section{The right half-plane model}

For some technical reasons we first transfer the study of the semigroup behavior from the open unit disk to right half-plane by using the Cayley transform

$$
C(z)=\frac{1+z}{1-z} .
$$

Now, given a semigroup $S=\left\{F_{t}\right\}_{t \geq 0} \subset \operatorname{Hol}(\Delta)$ with the Denjoy-Wolff point $\tau=1$, we construct the semigroup $\Sigma=\left\{\Phi_{t}\right\}_{t \geq 0}$ of holomorphic self-mappings of the right half-plane $\Pi=\{w \in \mathbb{C}: \operatorname{Re} w>0\}$ having the Denjoy-Wolff point at $\infty$ as follows:

$$
\Phi_{t}(w)=C \circ F_{t} \circ C^{-1}(w) .
$$

If $S$ is continuous (hence, differentiable) in $t$, then $\Sigma$ is too. More precisely, let $f$ be the infinitesimal generator of $S$. Then by (1.2), $f$ must be of the form $f(z)=-(1-z)^{2} p(z)$ with $\operatorname{Re} p(z) \geq 0, z \in \Delta$. Differentiating $\Phi_{t}$ given by (2.1) at $t=0^{+}$, we find that $\Sigma$ is generated by the mapping $-\phi$, where

$$
\phi(w)=2 p\left(C^{-1}(w)\right)
$$

(cf., [7, Lemma 3.7.1]). So, $\phi \in \operatorname{Hol}(\Pi, \bar{\Pi})$ and the semigroup $\Sigma=\left\{\Phi_{t}\right\}_{t \geq 0}$ satisfies the Cauchy problem

$$
\left\{\begin{array}{l}
\frac{\partial \Phi_{t}(w)}{\partial t}=\phi\left(\Phi_{t}(w)\right), \\
\left.\Phi_{t}(w)\right|_{t=0}=w, \quad w \in \Pi .
\end{array}\right.
$$

Concerning the Kønigs function $h$ defined by (1.4), one can modify it to $\sigma:=h \circ C^{-1}$. By direct calculations we check that this modified function satisfies Abel's functional equation

$$
\sigma\left(\Phi_{t}(w)\right)=\sigma(w)+t, \quad w \in \Pi,
$$

as well as the initial value problem:

$$
\sigma^{\prime}(w)=\frac{1}{\phi(w)}, \quad \sigma(1)=0 .
$$

It was already mentioned that the angular derivative $a=f^{\prime}(1)$ always exists. Furthermore, by the Berkson-Porta representation (1.2) with $\tau=1$ and formula (2.2), the function $\phi$ can be represented as follows:

$$
\phi(w)=a(w+1)+\varrho(w), \quad \text { where } \quad \angle \lim _{w \rightarrow \infty} \frac{\varrho(w)}{w}=0 .
$$


If, in addition, $f \in \mathcal{C}^{2}(1)$, that is, $f$ admits representation (1.6) with $a \geq 0$, then

$$
\phi(w)=a(w+1)-2 b+\varrho(w), \text { where } \lim _{w \rightarrow \infty} \varrho(w)=0 .
$$

Suppose now that the semigroup $S$ generated by $f$ is of parabolic type. Then we have $a=0$ in (1.6). So, formula (2.7) becomes

$$
\phi(w)=-2 b+\varrho(w) \text {, where } \lim _{w \rightarrow \infty} \varrho(w)=0 .
$$

In the case when $S$ is of parabolic type and $f \in \mathcal{C}^{3+\varepsilon}(1)$ for some $\varepsilon \geq 0$, we obtain in the same manner that

$$
\phi(w)=-2 b+\frac{4 c}{w+1}+\varrho(w), \text { where } \lim _{w \rightarrow \infty}(w+1)^{1+\varepsilon} \varrho(w)=0 .
$$

Since $\Sigma$ has the Denjoy-Wolff point at $\infty$, we have by Julia's Lemma (see, for example, [14, 15, 11]) that $\operatorname{Re} \Phi_{t}(w)$ is an increasing function in $t \geq 0$. The tangential convergence of the semigroup means that the function $\frac{\operatorname{Im} \Phi_{t}(w)}{\operatorname{Re} \Phi_{t}(w)}$ is unbounded as $t$ tends to infinity. Roughly speaking, the semigroup converges tangentially when $\left|\operatorname{Im} \Phi_{t}(w)\right|$ grows faster than $\operatorname{Re} \Phi_{t}(w)$. Moreover, the original semigroup $S=\left\{F_{t}\right\}_{t \geq 0}$ converges strongly tangentially if and only if the function $\operatorname{Re} \Phi_{t}(w)$ is bounded for each $w$ with $\operatorname{Re} w>0$. For this reason, strongly tangentially convergent semigroups were referred to in [4] as semigroups of finite shift; and weakly tangentially convergent semigroups as semigroups of infinite shift. Therefore, a semigroup $S$ converges strongly tangentially if and only if each trajectory of the semigroup $\Sigma$ defined by (2.1) has a vertical asymptote. More generally, a semigroup trajectory in the open unit disk has a finite limit curvature if and only if the corresponding trajectory in the right half-plane has an asymptote as $t \rightarrow \infty$.

Next we will consider hyperbolic and parabolic type semigroups in the right half-plane separately.

\section{The hyperbolic case}

Theorem 3.1 Let $\left\{\Phi_{t}\right\}_{t \geq 0} \in \operatorname{Hol}(\Pi)$ be a semigroup of hyperbolic type with the Denjoy-Wolff point at $\infty$ generated by mapping $-\phi$ with $\operatorname{Re} \phi(w) \geq$ $0, w \in \Pi$. Suppose that

$$
\phi(w)=\alpha w+\beta+\varrho(w), \text { where } \angle \lim _{w \rightarrow \infty} \varrho(w)=0 .
$$


Then for any initial point $w_{0} \in \Pi$, the trajectory $\left\{\Phi_{s}\left(w_{0}\right), s \geq 0\right\}$ has the asymptote defined by the following equality

$$
\operatorname{Im}[(\alpha w+\beta) \bar{B}]=0,
$$

where $B=\exp \left(\alpha\left(\sigma\left(w_{0}\right)-i \lim _{x \rightarrow \infty} \operatorname{Im} \sigma(x)\right)\right)$ and $\sigma$ is defined by (2.5).

Proof. Consider the semigroup $S=\left\{F_{t}\right\}_{t \geq 0}$ in the open unit disk defined by $F_{t}=C^{-1} \circ \Phi_{t} \circ C$. Comparing formulas (1.6), (2.7) and (3.1) we conclude that $S$ is generated by the mapping $f$ of the form (1.6) with $a=\alpha$ and $b=\frac{\alpha-\beta}{2}$. It was shown in [6, Theorem 1 and Remark 3] that in this case for each $t \geq 0$, the semigroup element $F_{t} \in \operatorname{Hol}(\Delta)$ is twice differentiable at the point $\tau=1$ and

$$
F_{t}^{\prime}(1)=e^{-t \alpha}, \quad F_{t}^{\prime \prime}(1)=\frac{\alpha-\beta}{\alpha} e^{-t \alpha}\left(e^{-t \alpha}-1\right) .
$$

Using (2.1), a direct calculation shows that

$$
\Phi_{t}(w)=e^{t \alpha} w+\frac{\beta}{\alpha}\left(e^{t \alpha}-1\right)+\Gamma(t, w),
$$

where $\lim _{w \rightarrow \infty} \Gamma(t, w)=0$. In particular, for each $w \in \Pi$ we have:

$$
\lim _{w \rightarrow \infty} \frac{\Phi_{t}(w)}{w}=e^{t \alpha}
$$

Furthermore, by (2.1) we have

$$
\lim _{t \rightarrow \infty} \arg \Phi_{t}(w)=-\lim _{t \rightarrow \infty} \arg \left(1-F_{t}\left(C^{-1}(w)\right)\right) .
$$

Therefore, formula (1.5) in Theorem 1.1 can be rewritten as

$$
\lim _{t \rightarrow \infty} \arg \Phi_{t}(w)=\alpha\left[\operatorname{Im} \sigma(w)-\lim _{x \rightarrow+\infty} \operatorname{Im} \sigma(x)\right] .
$$

Denote

$$
g_{t}(w)=\frac{\Phi_{t}(w)}{\left|\Phi_{t}(1)\right|}
$$

It follows by the continuous version of the Valiron theorem (see, for example, [18, 12]), that the limit $g(w)=\lim _{t \rightarrow \infty} g_{t}(w)$ exists for all $w \in \Pi$ and $g$ satisfies Schröder's functional equation

$$
g\left(\Phi_{s}(w)\right)=e^{s \alpha} g(w), \quad s \geq 0,
$$


which is actually equivalent to the differential equation

$$
g^{\prime}(w) \phi(w)=\alpha g(w)
$$

(cf., [15, 17]). In addition,

$$
\lim _{t \rightarrow \infty} \arg \Phi_{t}(w)=\arg g(w)
$$

and $g(1)=e^{-i \theta}$ for some $\theta \in \mathbb{R}$. Since $\sigma(1)=0$ (see formula (2.5)) and $g(w)=\lim _{t \rightarrow \infty} g_{t}(w)$, one calculates $\theta$ by using (3.5) and (3.6). Namely,

$$
\begin{aligned}
\theta & =-\arg g(1)=-\lim _{t \rightarrow \infty} \arg g_{t}(1)=-\lim _{t \rightarrow \infty} \arg \Phi_{t}(1) \\
& =-\alpha\left[\operatorname{Im} \sigma(1)-\lim _{x \rightarrow \infty} \operatorname{Im} \sigma(x)\right]=\alpha \lim _{x \rightarrow \infty} \operatorname{Im} \sigma(x) .
\end{aligned}
$$

To proceed we denote by $L_{t}\left(w_{0}\right)$ the tangent line to the trajectory $\left\{\Phi_{s}\left(w_{0}\right), s \geq 0\right\}$ at the point $\Phi_{t}\left(w_{0}\right)$ for a fixed $w_{0} \in \Pi$. Its equation is

$$
\operatorname{Im}\left[\left(w-\Phi_{t}\left(w_{0}\right)\right) \overline{\phi\left(\Phi_{t}\left(w_{0}\right)\right)}\right]=0,
$$

or, what is one and the same,

$$
\operatorname{Im}\left[w \overline{\phi\left(\Phi_{t}\left(w_{0}\right)\right)}\right]=\operatorname{Im}\left[\Phi_{t}\left(w_{0}\right) \overline{\phi\left(\Phi_{t}\left(w_{0}\right)\right)}\right] .
$$

Substituting (3.1) into (3.9) we see that

$$
\operatorname{Im}\left[w \overline{\left(\alpha \Phi_{t}\left(w_{0}\right)+\beta+\varrho\left(\Phi_{t}\left(w_{0}\right)\right)\right)}\right]=\operatorname{Im}\left[\Phi_{t}\left(w_{0}\right) \overline{\left(\beta+\varrho\left(\Phi_{t}\left(w_{0}\right)\right)\right)}\right] .
$$

Dividing now equation (3.10) by $\left|\Phi_{t}(1)\right|$, we have

$$
\begin{array}{r}
\operatorname{Im}\left[w \cdot \overline{\left(\alpha g_{t}\left(w_{0}\right)+\frac{\beta}{\left|\Phi_{t}(1)\right|}+\frac{\varrho\left(\Phi_{t}\left(w_{0}\right)\right)}{\left|\Phi_{t}(1)\right|}\right)}\right] \\
=\operatorname{Im}\left[g_{t}\left(w_{0}\right) \overline{\left(\beta+\varrho\left(\Phi_{t}\left(w_{0}\right)\right)\right)}\right] .
\end{array}
$$

Letting $t$ tend to infinity, we conclude that the net of lines $\left\{L_{t}\left(w_{0}\right)\right\}$ converges to the limit line $L\left(w_{0}\right)$ the equation of which by (3.11) is

$$
\alpha \operatorname{Im}\left[w \overline{g\left(w_{0}\right)}\right]=\operatorname{Im}\left[g\left(w_{0}\right) \bar{\beta}\right]
$$


or

$$
\operatorname{Im}\left[(\alpha w+\beta) \overline{g\left(w_{0}\right)}\right]=0,
$$

Comparing now (2.5) with (3.7), we get

$$
g(w)=\exp \left(\alpha\left(\sigma(w)-i \lim _{x \rightarrow \infty} \operatorname{Im} \sigma(x)\right)\right) .
$$

Setting $B=g\left(w_{0}\right)$, we complete the proof.

Proof of Theorem 1.2. Suppose that the generator $f$ of a semigroup $S$ is of the form (1.6). Construct the semigroup $\Sigma$ in the right half-plane and denote its generator by $-\phi$. Then $\phi$ has form (3.1) with $\alpha=a$ and $\beta=a-2 b$. We have already shown that each semigroup trajectory $\left\{\Phi_{t}\left(w_{0}\right), t \geq 0\right\}$ has the asymptote defined by (3.2). Substituting in this formula $\alpha, \beta$ and $w=\frac{1+z}{1-z}$, we attain at (1.7).

\section{The parabolic case}

Theorem 4.1 Let $\left\{\Phi_{t}\right\}_{t \geq 0} \in \operatorname{Hol}(\Pi)$ be a semigroup of parabolic type with the Denjoy-Wolff point at $\infty$ generated by mapping $-\phi$.

(i) Suppose that $\phi$ admits the representation

$$
\phi(w)=\beta+\varrho(w)
$$

where $\varrho \in \operatorname{Hol}(\Pi, \mathbb{C}), \lim _{z \rightarrow \infty} \varrho(w)=0$. Then

$$
\Phi_{t}(w)=\beta t+\Gamma(w, t), \quad \text { where } \lim _{t \rightarrow \infty} \frac{\Gamma(w, t)}{t}=0
$$

and

$$
\lim _{t \rightarrow \infty}\left(\Phi_{t}(w)-\Phi_{t}(1)\right)=\beta \sigma(w) .
$$

(ii) If $\beta \neq 0$ and $\varrho$ in 4.1 is of the form $\varrho(w)=\frac{\gamma}{w+1}+\varrho_{1}(w)$ with $\varrho_{1} \in \operatorname{Hol}(\Pi, \mathbb{C}), \lim _{w \rightarrow \infty} w \varrho(w)=0$, i.e., $\phi$ admits the representation

$$
\phi(w)=\beta+\frac{\gamma}{w+1}+\varrho_{1}(w),
$$

then

$$
\Phi_{t}(w)=\beta t+\frac{\gamma}{\beta} \log (t+1)+\Gamma_{1}(w, t),
$$


where $\lim _{t \rightarrow \infty} \frac{\Gamma_{1}(w, t)}{\log (t+1)}=0$, and

$$
\lim _{t \rightarrow \infty} t\left(\Phi_{t}(w)-\Phi_{t}(1)-\beta \sigma(w)\right)=\frac{\gamma \sigma(w)}{\beta}
$$

(iii) If function $\varrho_{1}$ in 4 4.4) satisfies the condition $\lim _{w \rightarrow \infty} w^{1+\varepsilon} \varrho_{1}(w)=0$ for some $\varepsilon>0$, then there is a constant $A$ such that for all $w \in \Pi$

$$
\Phi_{t}(w)=\beta t+\frac{\gamma}{\beta} \log (t+1)+\beta \sigma(w)+A+\Gamma_{2}(w, t),
$$

where $\lim _{t \rightarrow \infty} \Gamma_{2}(w, t)=0$.

Proof. (i) Fix $w \in \Pi$ and consider $\Phi_{t}(w)$ as a (complex valued) function of the real variable $t$. This function tends to infinity as $t \rightarrow \infty$. Thus, by the L'Hospital rule

$$
\lim _{t \rightarrow \infty} \frac{\Phi_{t}(w)}{t}=\lim _{t \rightarrow \infty} \frac{\phi\left(\Phi_{t}(w)\right)}{1}=\beta .
$$

Furthermore,

$$
\begin{array}{r}
\lim _{t \rightarrow \infty}\left(\Phi_{t}(w)-\Phi_{t}(1)\right)=\lim _{t \rightarrow \infty} \int_{1}^{w}\left(\Phi_{t}(z)\right)^{\prime} d z=\lim _{t \rightarrow \infty} \int_{1}^{w} \frac{\phi\left(\Phi_{t}(z)\right)}{\phi(z)} d z \\
=\lim _{t \rightarrow \infty} \int_{1}^{w} \frac{\beta+\varrho\left(\Phi_{t}(z)\right)}{\phi(z)} d z=\beta \int_{1}^{w} \frac{d z}{\phi(z)}=\beta \sigma(w) .
\end{array}
$$

(ii) To prove this assertion we need to show that

$$
\lim _{t \rightarrow \infty} \frac{1}{\log (t+1)} \cdot\left(\Phi_{t}(w)-\beta t-\frac{\gamma}{\beta} \log (t+1)\right)=0 .
$$

To do this, we calculate

$$
\begin{aligned}
& \Phi_{t}(w)-\beta t-\frac{\gamma}{\beta} \log (t+1)=\int_{0}^{t}\left(\Phi_{s}(w)-\beta s-\frac{\gamma}{\beta} \log (s+1)\right)^{\prime} d s+w \\
& =\int_{0}^{t}\left(\phi\left(\Phi_{s}(w)\right)-\beta-\frac{\gamma}{\beta(s+1)}\right) d s+w \\
& =\int_{0}^{t}\left(\frac{\gamma}{\Phi_{s}(w)+1}-\frac{\gamma}{\beta(s+1)}+\varrho_{1}\left(\Phi_{s}(w)\right)\right) d s+w .
\end{aligned}
$$


Note that

$$
\begin{aligned}
& \frac{\gamma}{\Phi_{s}(w)+1}-\frac{\gamma}{\beta(s+1)}+\varrho_{1}\left(\Phi_{s}(w)\right) \\
& =\frac{1}{s+1} \cdot\left(\frac{\gamma(s+1)}{\Phi_{s}(w)+1}-\frac{\gamma}{\beta}+\frac{\Phi_{s}(w) \varrho_{1}\left(\Phi_{s}(w)\right)}{\Phi_{s}(w) /(s+1)}\right),
\end{aligned}
$$

where by (i) and our assumption the second factor tends to zero. Therefore, for each $\varepsilon>0$ there is $t_{0}$ such that

$$
\left|\frac{\gamma(s+1)}{\Phi_{s}(w)+1}-\frac{\gamma}{\beta}+\frac{\Phi_{s}(w) \varrho\left(\Phi_{s}(w)\right)}{\Phi_{s}(w) /(s+1)}\right|<\frac{\varepsilon}{2}
$$

for $s>t_{0}$. Hence

$$
\begin{aligned}
& \left|\int_{0}^{t}\left(\frac{\gamma}{\Phi_{s}(w)+1}-\frac{\gamma}{\beta(s+1)}+\varrho_{1}\left(\Phi_{s}(w)\right)\right) d s\right| \leq \\
\leq \quad & \left|\int_{0}^{t_{0}}\left(\frac{\gamma}{\Phi_{s}(w)+1}-\frac{\gamma}{\beta(s+1)}+\varrho_{1}\left(\Phi_{s}(w)\right)\right) d s\right|+\int_{t_{0}}^{t} \frac{\varepsilon / 2}{s+1} d s .
\end{aligned}
$$

Now for $t_{0}$ given above, choose $t_{1}$ such that

$$
\left|\int_{0}^{t_{0}}\left(\frac{\gamma}{\Phi_{s}(w)+1}-\frac{\gamma}{\beta(s+1)}+\varrho_{1}\left(\Phi_{s}(w)\right)\right) d s\right| \leq \frac{\varepsilon}{2} \log \left(t_{1}+1\right) .
$$

So, for $t \geq \max \left(t_{0}, t_{1}\right)$ we have

$$
\begin{aligned}
& \left|\int_{0}^{t}\left(\frac{\gamma}{\Phi_{s}(w)+1}-\frac{\gamma}{\beta(s+1)}+\varrho_{1}\left(\Phi_{s}(w)\right)\right) d s\right| \leq \\
& \leq \frac{\varepsilon}{2} \log \left(t_{1}+1\right)+\int_{t_{0}}^{t} \frac{\varepsilon / 2}{s+1} d s \leq \varepsilon \log (t+1) .
\end{aligned}
$$

Since $\varepsilon>0$ can be chosen arbitrarily, we conclude that the limit in (4.8), hence, in (4.5), is zero. In addition, we have that

$$
\begin{aligned}
& \lim _{t \rightarrow \infty} t\left(\Phi_{t}(w)-\Phi_{t}(1)-\beta \sigma(w)\right)=\lim _{t \rightarrow \infty} t \cdot \int_{1}^{w}\left(\Phi_{t}(z)-\beta \sigma(z)\right)^{\prime} d z \\
= & \lim _{t \rightarrow \infty} t \cdot \int_{1}^{w} \frac{\phi\left(\Phi_{t}(z)\right)-\beta}{\phi(z)} d z=\lim _{t \rightarrow \infty} t \cdot \int_{1}^{w} \frac{\frac{\gamma}{\Phi_{t}(z)}+\varrho_{1}\left(\Phi_{t}(z)\right)}{\phi(z)} d z \\
= & \lim _{t \rightarrow \infty} \int_{1}^{w} \frac{t}{\Phi_{t}(z)} \cdot \frac{\gamma+\Phi_{t}(z) \varrho_{1}\left(\Phi_{t}(z)\right)}{\phi(z)} d z=\frac{\gamma \sigma(w)}{\beta} .
\end{aligned}
$$


This proves (4.6).

(iii) First, we prove that for each $w \in \Pi$ the limit

$$
H(w):=\lim _{t \rightarrow \infty}\left(\Phi_{t}(w)-\beta t-\frac{\gamma}{\beta} \log (t+1)\right)
$$

exists. Indeed,

$$
\begin{aligned}
& \Phi_{t}(w)-\beta t-\frac{\gamma}{\beta} \log (t+1) \\
& =\int_{0}^{t} \frac{d}{d s}\left(\Phi_{s}(w)-\beta s-\frac{\gamma}{\beta} \log (s+1)\right) d s+w \\
& =\int_{0}^{t}\left(\phi\left(\Phi_{s}(w)\right)-\beta-\frac{\gamma}{\beta(s+1)}\right) d s+w \\
& =\int_{0}^{t} \varrho_{1}\left(\Phi_{s}(w)\right) d s+\frac{\gamma}{\beta} \int_{0}^{t}\left(\frac{\beta}{\Phi_{s}(w)+1}-\frac{1}{s+1}\right) d s+w .
\end{aligned}
$$

To this end, we need to show that the two integrals

$$
\int_{0}^{\infty} \varrho_{1}\left(\Phi_{s}(w)\right) d s \text { and } \int_{0}^{\infty}\left(\frac{\beta}{\Phi_{s}(w)+1}-\frac{1}{s+1}\right) d s
$$

converge. Writing the first integral in the form

$$
\int_{0}^{\infty} \frac{\Phi_{s}^{1+\varepsilon}(w) \varrho_{1}\left(\Phi_{s}(w)\right)}{\Phi_{s}^{1+\varepsilon}(w)} d s,
$$

we see that it converges since the nominator tends to zero and the denominator behaves as $s^{1+\varepsilon}$ when $s \rightarrow \infty$ by (4.2).

Consider the second integral. Using (4.5), we have:

$$
\begin{aligned}
& \int_{0}^{\infty} \frac{\beta(s+1)-\Phi_{s}(w)-1}{\left(\Phi_{s}(w)+1\right)(s+1)} d s \\
& =\int_{0}^{\infty} \frac{\beta-1-\frac{\gamma}{\beta} \log (s+1)-\Gamma_{1}(w, s)}{\left(\beta s+\frac{\gamma}{\beta} \log (s+1)+\Gamma(w, s)+1\right)(s+1)} d s \\
& =\int_{0}^{\infty} \frac{1}{(s+1)^{2}} \cdot \frac{\beta-1-\frac{\gamma}{\beta} \log (s+1)-\Gamma_{1}(w, s)}{\beta-\frac{1-\beta}{s+1}+\frac{\gamma \log (s+1)}{\beta(s+1)}+\frac{\Gamma_{1}(w, s)}{s+1}} d s .
\end{aligned}
$$

Since the second factor in the integrand is $O(\log (s+1))$, this integral also converges. 
Finally, by (4.3) we get

$$
H(w)-H(1)=\lim _{t \rightarrow \infty}\left(\Phi_{t}(w)-\Phi_{t}(1)\right)=\beta \sigma(w) .
$$

This proves formula (4.7) with $A=H(1)$ and $\Gamma_{2}(w, t)=\Phi_{t}(w)-\beta t-$ $\frac{\gamma}{\beta} \log (t+1)-\beta \sigma(w)-A$. The proof of the theorem is complete.

Theorem 4.2 Let $\left\{\Phi_{t}\right\}_{t \geq 0} \in \operatorname{Hol}(\Pi)$ be a semigroup of parabolic type generated by mapping $-\phi$, where $\phi$ has the form

$$
\phi(w)=\beta+\frac{\gamma}{w+1}+\varrho_{1}(w)
$$

with $\varrho_{1} \in \operatorname{Hol}(\Pi, \mathbb{C}), \beta, \gamma \in \mathbb{C}$. Assume that $\beta \neq 0$ and $\lim _{z \rightarrow \infty} w^{1+\varepsilon} \varrho_{1}(w)=$ 0 for some $\varepsilon>0$. The following assertions hold.

(a) If $\operatorname{Im} \frac{\gamma}{\beta^{2}} \neq 0$, then for any trajectory $\left\{\Phi_{t}(w), t \geq 0\right\}$ there is no asymptote.

(b) Otherwise, if $\operatorname{Im} \frac{\gamma}{\beta^{2}}=0$, then each trajectory $\left\{\Phi_{t}(w), t \geq 0\right\}$ has an asymptote. In this case, the asymptote equation is $\operatorname{Im}[w \cdot \bar{\beta}]=B$ with

$$
B=|\beta|^{2} \operatorname{Im} \sigma(w)+\int_{0}^{\infty} \operatorname{Im}\left(\phi\left(\Phi_{s}(1)\right) \bar{\beta}-\frac{\gamma \bar{\beta}}{\beta(s+1)}\right) d s-\operatorname{Im} \beta .
$$

Proof. It follows by Theorem 4.1 (i) that

$$
\lim _{t \rightarrow \infty} \arg \Phi_{t}(w)=\lim _{t \rightarrow \infty} \arg \frac{\Phi_{t}(w)}{t}=\arg \beta
$$

So, if an asymptote exists, its equation must be $\operatorname{Im}[w \cdot \bar{\beta}]=$ const.

(a) If $\operatorname{Im} \frac{\gamma}{\beta^{2}} \neq 0$, then by (4.5) we have

$$
\begin{aligned}
\operatorname{Im}\left[\Phi_{t}(w) \cdot \bar{\beta}\right] & =\operatorname{Im}\left[\left(\beta t+\frac{\gamma}{\beta} \log (t+1)+\Gamma(w, t)\right) \cdot \bar{\beta}\right] \\
& =\log (t+1)|\beta|^{2} \operatorname{Im} \frac{\gamma}{\beta^{2}}+\operatorname{Im}[\Gamma(w, t) \cdot \bar{\beta}]
\end{aligned}
$$

Thus, under our assumption we have that $\operatorname{Im}\left[\Phi_{t}(w) \cdot \bar{\beta}\right]$ is unbounded, so there is no asymptote for the trajectory $\left\{\Phi_{t}, t \geq 0\right\}, w \in \Pi$. 
(b) If $\operatorname{Im} \frac{\gamma}{\beta^{2}}=0$, then by (4.7) we obtain that $\operatorname{Im}\left[\Phi_{t}(w) \cdot \bar{\beta}\right]=\operatorname{Im}\left[\left(\beta t+\frac{\gamma}{\beta} \log (t+1)+\beta \sigma(w)+A+\Gamma_{2}(w, t)\right) \cdot \bar{\beta}\right]$,

where $\lim _{t \rightarrow \infty} \Gamma_{2}(w, t)=0$. Hence,

$$
\lim _{t \rightarrow \infty} \operatorname{Im}\left[\Phi_{t}(w) \cdot \bar{\beta}\right]=|\beta|^{2} \operatorname{Im} \sigma(w)+\operatorname{Im}(A \bar{\beta})
$$

So, in this case the asymptote for the trajectory $\left\{\Phi_{t}(w), t \geq 0\right\}$ does exist. But we have already seen in the proof of Theorem 4.1 that

$$
A=H(1)=\int_{0}^{\infty}\left(\phi\left(\Phi_{s}(1)\right)-\beta-\frac{\gamma}{\beta(s+1)}\right) d s+1
$$

and this completes our proof.

Again we observe that Theorems 1.3 and 1.4 are interpretations of Theorems 4.2 and 4.1, respectively, for the semigroup $S=\left\{F_{t}\right\}_{t \geq 0}, F_{t}=$ $C^{-1} \circ \Phi_{t} \circ C$, acting in the open unit disk.

Proof of Theorem 1.5. Assume that

$$
\lim _{t \rightarrow \infty} \operatorname{Im}\left[\bar{b}\left(\frac{1}{1-F_{t}(z)}-\frac{1}{1-G_{t}(z)}\right)\right]=0
$$

Using Theorem 1.4 (iii), we have

$$
\begin{aligned}
& \lim _{t \rightarrow \infty} \operatorname{Im}\left[\bar{b}\left(\frac{1}{1-F_{t}(z)}-\frac{1}{1-G_{t}(z)}\right)\right]= \\
& \lim _{t \rightarrow \infty} \operatorname{Im}\left[\frac{\left(c_{2}-c_{1}\right) \bar{b}}{b} \log (t+1)+|b|^{2}\left(h_{2}(z)-h_{1}(z)\right)+\bar{b}\left(A_{1}-A_{2}\right)\right]
\end{aligned}
$$

where $A_{1}, A_{2}$ are constants and $h_{1}, h_{2}$ are Kœnigs functions for $\left\{F_{t}\right\}_{t \geq 0}$ and $\left\{G_{t}\right\}_{t \geq 0}$, respectively. The last limit may be zero only if $\operatorname{Im} \frac{\left(c_{2}-c_{1}\right) \bar{b}}{b}=0$ and then $\operatorname{Im}\left(h_{1}(z)-h_{2}(z)\right)=\operatorname{Im} \frac{A_{1}-A_{2}}{b}$. Therefore, $h_{1}(z)-h_{2}(z)=$ const. Since $h_{1}(0)=h_{2}(0)=0$, we have $h_{1}(z)=h_{2}(z)$. Now by (1.4), we conclude that $f \equiv g$.

For the sake of completeness we provide an equivalent result for the right half-plane case. 
Theorem 4.3 Let $\left\{\Phi_{t}\right\}_{t \geq 0},\left\{\Psi_{t}\right\}_{t \geq 0} \subset \operatorname{Hol}(\Pi)$ be two continuous semigroups of holomorphic self-mappings in the right half-plane generated by mappings $-\phi$ and $-\psi$, respectively. Suppose that $\phi$ and $\psi$ admit the following representations:

$$
\begin{gathered}
\phi(w)=\beta+\frac{\gamma}{w+1}+\varrho(w), \\
\psi(w)=\beta+\frac{\gamma_{1}}{w+1}+\varrho_{1}(w),
\end{gathered}
$$

with $\varrho, \varrho_{1} \in \operatorname{Hol}(\Pi, \mathbb{C}), \lim _{z \rightarrow \infty} w^{1+\varepsilon} \varrho(w)=\lim _{z \rightarrow \infty} w^{1+\varepsilon} \varrho_{1}(w)=0, \beta, \gamma, \gamma_{1} \in$ $\mathbb{C}$, and let $\beta \neq 0$. If

$$
\lim _{t \rightarrow \infty} \operatorname{Im}\left[\bar{\beta}\left(\Phi_{t}(w)-\Psi_{t}(w)\right)\right]=0,
$$

then the semigroups coincide.

Acknowledgments. This research is part of the European Science Foundation Networking Programme HCAA. The second author is supported by the European Community project TODEQ (MTKD-CT-2005030042).

\section{References}

[1] E. Berkson and H. Porta, Semigroups of analytic functions and composition operators, Michigan Math. J. 25 (1978), 101-115.

[2] P. S. Bourdon and J. H. Shapiro, Cyclic phenomena for composition operators, Mem. Amer. Math. Soc. 125 (1997), no. 596.

[3] M. D. Contreras and S. Díaz-Madrigal, Analytic flows on the unit disk: angular derivatives and boundary fixed points, Pacific $J$. Math. 222 (2005), 253-286.

[4] M. D. Contreras, S. Díaz-Madrigal and Ch. Pommerenke, Second angular derivatives and parabolic iteration in the unit disk, Trans. Amer. Math. Soc. 362 (2010), 357-388.

[5] M. Elin, D. Khavinson, S. Reich and D. Shoikhet, Linearization models for parabolic dynamical systems via Abel's functional equation, Ann. Acad. Sci. Fen. 35 (2010), 1-34 
[6] M. Elin, M. Levenshtein, S. Reich and D. Shoikhet, Commuting semigroups of holomorphic mappings, Math. Scand. 103 (2008), $295-319$.

[7] M. Elin, S. Reich and D. Shoikhet, Complex Dynamical Systems and the Geometry of Domains in Banach Spaces, Dissertationes Math. (Rozprawy Mat.) 427 (2004), 62 pp.

[8] M. Elin, S. Reich, D. Shoikhet and F. Yacobzon, Asymptotic behavior of one-parameter semigroups and rigidity of holomorphic generators, Complex Anal. Oper. Theory 2 (2008), 55-86.

[9] M. Elin and D. Shoikhet, Dynamic extension of the Julia-WolffCarathéodory theorem, Dynam. Systems Appl. 10 (2001), 421-438.

[10] M. Elin and D. Shoikhet, Angle distortion theorems for starlike and spirallike functions with respect to a boundary point, Intern. Journ. Math. Math. Sci., 2006, Article ID 81615, 1-13.

[11] M. Elin and D. Shoikhet, Linearization Models for Complex Dynamical Systems. Topics in univalent functions, functions equations and semigroup theory, Birkhäuser Basel, 2010.

[12] M. Elin, D. Shoikhet and V. Volkovich, Semigroups of holomorphic mappings on the unit disk with a boundary fixed point, Int. J. Pure Appl. Math. 12 (2004), 427-453.

[13] M. Elin, D. Shoikhet and F. Yacobzon, Linearization models for parabolic type semigroups, J. Nonlinear Convex Anal. 9 (2008), 205-214.

[14] J. H. Shapiro, Composition Operators and Classical Function Theory, Springer, Berlin, 1993.

[15] D. Shoikhet, Semigroups in Geometrical Function Theory, Kluwer, Dordrecht, 2001.

[16] D. Shoikhet, Another look at the Burns-Krantz theorem, J. Anal. Math. 105 (2008), 19-42.

[17] A. Siskakis, Semigroups of composition operators on spaces of analytic functions, a review. Studies on composition operators (Laramie, WY, 1996), 229-252, Contemp. Math. 213 Amer. Math. Soc., Providence, RI, 1998. 
[18] G. Valiron, Fonctions Analytiques, Presses Univ. France, Paris, 1954. 